\title{
Analysis of Interference on Mirror-Aided Non-LOS Backhaul Data Transmission in VLC Attocell Networks
}

\author{
Yuhui Wu* · Mario Pickavet · Didier Colle
}

Received: date / Accepted: date

\begin{abstract}
Visible light communication (VLC) is a promising technology that addresses the bandwidth bottleneck of radio-frequency based indoor wireless networks. Optical attocell networks using VLC backhaul links have been proposed. Line-of-sight (LOS) and mirror-aided non-LOS links can be used for backhaul transmission. As the visible light spectrum is used for both access downlink and bidirectional backhaul transmission, co-channel interference is a major issue. In this paper, we analyze the performance of optical attocell networks using VLC backhaul links taking into account illumination and eye safety requirements. Based on the signal-to-interference-plus-noise ratio (SINR) of different backhaul links, the bandwidth allocation ratio when using an in-band frequency reused scheme is discussed. Finally, aggregate data rates of networks using different VLC backhaul configurations are compared. Results show that LOS backhaul configuration can achieve nearly optimal network performance while mirror-aided non-LOS backhaul configuration is more robust against misalignment.
\end{abstract}

Keywords Visible light communication · backhaul network $\cdot$ co-channel interference

\section{Introduction}

Due to the increasing number of mobile devices and the growing demand on data traffic for indoor activities such as virtual reality and high-definition video streaming, radiofrequency (RF) based indoor wireless networks are experiencing spectrum crisis. Visible light communication (VLC)

Yuhui Wu

Ghent University-IMEC, Technologiepark 126, B-9052 Gent (Zwijnaarde)

Tel.: +3293314977

E-mail: yuhui.wu@ugent.be
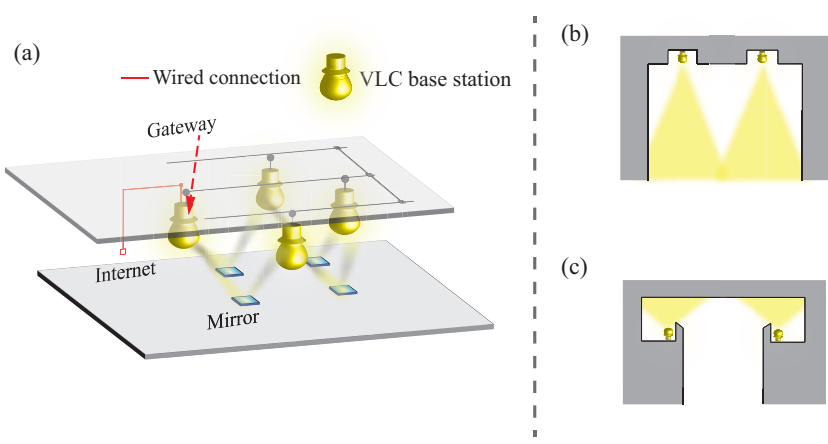

Fig. 1 (a) Mirror-aided non-LOS VLC link-based backhaul network; (b)Illumination design with embedded ceiling lamps; (c) Illumination design with diffuse lighting system

using off-the-shelf light-emitting-diodes (LEDs) and photodiodes (PDs) as front-end devices is an emerging technology to address the bandwidth bottleneck of RF technologies [1]. A VLC system uses intensity modulation and direct detection (IM/DD) which represents the transmitted signal as high-frequency fluctuation in the radiant intensity. Thus, VLC supports simultaneous illumination and data transmission. As LED lamps are ubiquitous and IM/DD technology is simple and low-cost, a VLC-based optical attocell network is a cost-efficient solution that complements the spectrum shortage of current wireless home networks [2-5].

In a VLC-based optical attocell network, each LED lamp is a base station (BS) which covers mobile devices in a small cell with radius ranging from one to several meters. An access network is composed of communication links amongst user equipment (UE) and BSs. Access downlinks from BSs to UEs are based on VLC, while uplinks normally use infrared to carry information. BSs communicate with each other through a backhaul network. The backhaul network is connected to the Internet through a gateway which can be one of the VLC BSs. Although there is growing interest on access downlink 
transmission in recent years [6-8], the capacity of backhaul links is not considered in those studies. There is a number of backhaul solutions proposed for optical attocell networks. Wired approaches such as power-line communication (PLC) and power-over-Ethernet $(\mathrm{PoE})$ which provide simultaneous power supply and data transmission have been considered in $[9,10]$. These wired backhaul solutions require installing additional control devices like modems or replacing current power cables. Thus, the cost for installation and maintenance could be extremely high. In addition, PLC is vulnerable to RF interference and disturbance on power-lines, as unshielded wirings may turn to unintended antennas [11]. Data rates of PLC devices are reduced due to noises and appliance interference [12]. A backhaul approach using millimeterwave (mmWave) has been considered in [13]. Although the mmWave-based backhaul network has the potential for highspeed data transmission, the bio-effects on human bodies caused by non-ionizing mmWave radiation deserve consideration and further study [14]. Security is also an issue when using mmWave as the mmWave signal can go through walls. In [15], a VLC link-based backhaul solution using line-ofsight (LOS) links between auxiliary LEDs and PDs has been proposed. The downlink performance of a two-tier optical attocell network using LOS VLC backhaul links has been analyzed in [16]. It has been shown that LOS VLC backhaul links attain good performance especially when the frequency band is fully reused. However, a significant drawback of the LOS VLC backhaul solution is the strict LOS requirement. Imperfect alignment or obstruction may cause performance degradation due to signal power reduction and larger interference from neighboring transmitters. As there are different interior lighting design strategies, the LOS condition may not be fulfilled in some applications where lamps are placed at different heights or separated by walls. In order to improve the generality of VLC backhaul links, we propose to use mirror-aided non-LOS VLC backhaul links as an alternative solution. A network based on mirror-aided non-LOS backhaul links is illustrated in Fig. 1(a). Mirrors or glossy floor tiles are used to provide specular reflections. The advantage of this backhaul solution is that non-LOS configuration can be used in indoor applications where the LOS condition may not be fulfilled, for instance, interior illumination design like embedded lamps and diffuse lighting shown in Fig. 1(b) and (c), respectively. Specular reflections are directional and the reflectivity of glossy surfaces and mirrors is higher than that of diffuse surfaces. Thus, the quality of mirror-aided nonLOS links is better than that of normal diffuse non-LOS links [17]. The position of mirrors is determined by locations and orientations of LED lamps. In order to mitigate multi-path effects, small mirrors are preferred [17]. When mirrors are placed on the floor, obstructions caused by surroundings and moving people could temporarily degrade the performance of non-LOS links. This can be solved in higher layers (network layer) by using adjacent BSs as relays.

Similar to the access network, co-channel interference is a major issue that affects the performance of the backhaul network. Interference of an optical attocell network using LOS-VLC backhaul links has been analyzed in [16]. Two frequency reused schemes, fully reused (FR) and in-band (IB) methods, have been discussed. However, the analysis of co-channel interference only considered downlink transmission, i.e. transmission from the gateway to UEs. In this paper, we analyze the performance of the proposed backhaul solution in a two-tier optical attocell network. In the analysis a shortest-path routing algorithm is used such that the backhaul network has a tree structure. The interference caused by backhaul uplink transmission, i.e., backhaul data sent from tier-2 to tier-1 BSs and from tier-1 BSs to the gateway, is also taken into account. The channel performance is assessed in terms of signal-to-interference-plus-noise ratio (SINR). DC biased optical orthogonal frequency division multiplexing (DCO-OFDM) is considered due to its low complexity of equalization and high spectral efficiency [18]. Network aggregate data rates using different VLC backhaul solutions are calculated and compared.

\section{Physical layer channel model}

In a VLC system, data transmitted by the LED is modulated using IM. The PD of the receiving device converts the optical power to electrical signal. The channel model described in the following subsection can be used to simulate the gain of LOS and mirror-aided non-LOS VLC links. Multi-bounce reflections are neglected as the LOS component and the first-bounce specular reflection component dominate impulse responses of LOS links and mirror-aided nonLOS links, respectively [18] [17]. Despite the simplicity and cost-efficiency of IM/DD, the channel capacity is limited by the modulation bandwidth of LEDs. In addition to the channel model, the modulation scheme used for IM/DD signal has to be considered. Optical OFDM is a viable modulation scheme for VLC systems due to its high spectral efficiency. High peak-to-average-power-ratio of optical OFDM modulated signal, which is the major issue of using OFDM in RF systems, provides higher electrical signal-to-noise ratio (SNR) for VLC systems when the average transmitted optical power is limited by illumination requirements. Amongst optical OFDM schemes proposed for IM/DD VLC systems, DCO-OFDM which has higher spectral efficiency and lower complexity is considered in this work. We assume that all LEDs are Lambertian point sources and operate within the linear dynamic range such that non-linear distortions are avoided. Thermal noise and shot noise at the receiver are modeled as additive white Gaussian noises (AWGN). 
(a)

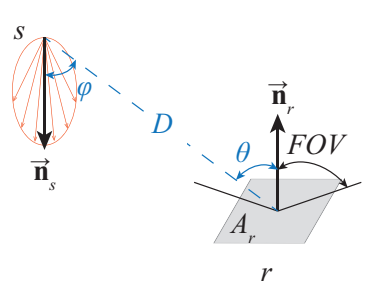

(b)

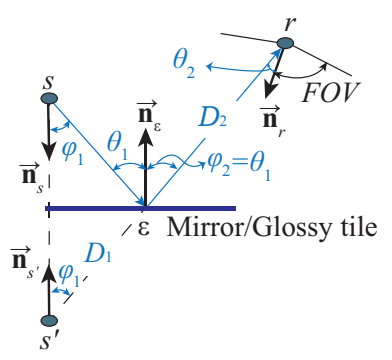

result should be multiplied by the reflectivity of the mirror. Here, $s^{\prime}$ is the mirror image of the sender $s$. The condition of such a mirror-aided non-LOS link is that there isn't obstruction between the sender and the mirror and between the mirror and the receiver. In addition, the receiver's FOV must be large enough to "see" the reflecting point $\varepsilon$. In order to simplify notations, we use $G^{L O S}$ to denote the gain of a LOS path and $G^{m a-n L O S}$ to denote the gain of a mirror-obstructed LOS path, i.e. a mirror-aided non-LOS path.

Fig. 2 Geometry of (a) a LOS path from a Lambertian sender $s$ to a receiver $r, \vec{n}_{s}$ and $\vec{n}_{r}$ represent orientations of the sender and the receiver, respectively, the red lobe represents the emission pattern of the sender; (b) a mirror-obstructed LOS path from a virtual sender $s^{\prime}$ (the mirror image of a sender $s$ ) to a receiver $r$

\subsection{Light propagation model}

Fig. 2 (a) illustrates the geometry of a LOS VLC link from a Lambertian sender $s$ (LED) to the PD of a receiver $r$. The emission pattern of a LED can be described by a Lambert order $m=-\ln 2 / \ln \left(\cos \left(\Phi_{1 / 2}\right)\right)$, where $\Phi_{1 / 2}$ is the semi-angle at half-intensity of the LED emission pattern. The gain of the LOS path $G_{s, r}$ is the ratio of the received optical power to the transmitted optical power. This can be calculated with a normalized Lambertian pattern [19]

$G_{s, r}=\frac{(m+1) A_{r}}{2 \pi D^{2}} \cos ^{m}(\phi) \cos (\theta) \operatorname{rect}\left(\frac{\theta}{\Theta}\right)$,

where $\phi$ and $\theta$ are angles of LOS paths measured from orientations of the sender $s$ and the receiver $r$, respectively, $D$ is the Euclidean distance from $s$ to $r, A_{r}$ is the active area of the $\mathrm{PD}, \Theta$ is the field-of-view (FOV) of the PD. The rectangular function $\operatorname{rect}(x)$ is 1 if $|x| \leq 1$, and 0 otherwise.

A non-LOS link implies that $G_{s, r}$ between the transmitting $B S_{S}$ and the receiving $B S_{r}$ is 0 . The non-LOS channel has lower gains than a channel with a dominant LOS component when all light rays are diffusely reflected from Lambertian surfaces [20]. Nonetheless, specular reflections from glossy surfaces or mirrors are directional. In order to improve the channel performance, we propose to add a dominant first-bounce specularly reflected component to the non-LOS channel impulse response. This can be done by polishing the floor or replacing some tiles on the floor with glossy tiles or mirrors. In order to simplify the simulation, specular reflections from glossy surfaces are emulated by placing perfect mirrors on the floor. We assume that all mirrors are ideal such that each incident ray is perfectly reflected towards the direction defined by the law of reflection. In order to simplify calculations of gains of mirror-aided non-LOS links, we model the specular reflection as a mirror-obstructed LOS path as shown in Fig. 2 (b). The calculation of the gain of the mirror-obstructed LOS path $G_{S^{\prime}, r}$ is similar to (1) by substituting $\phi$ with $\phi_{1}, \theta$ with $\theta_{2}$, and $D$ with $\left(D_{1}+D_{2}\right)$. The final

\subsection{Optical OFDM}

Unlike RF technologies, intensity modulated OFDM signals transmitted in VLC channels must be real and non-negative. Data symbols are quadrature amplitude modulated (QAM) to represent the magnitude of the transmitted signal in the frequency domain. The time-domain signal is the inverse discrete Fourier transform of the modulated data symbols and represented by optical power transmitted by LEDs. Modulated data symbols of all subcarriers in the frequency domain are denoted by $X_{n}, n=0,1, \cdots, N-1$, where $N$ is the number of OFDM subcarriers. The 0 -th and $(N / 2)$-th subcarriers are set to zero, where the $(N / 2)$-th subcarrier is the Nyquist term. Hermitian symmetry $X_{n}=X_{N-n}^{*}$ is necessary to ensure real time-domain signals, where $[\cdot]^{*}$ denotes the complex conjugate operation. In DCO-OFDM, DC-bias is added to the modulated signal and all negative values are clipped to ensure non-negative optical intensity. The clipping noise can be neglected when choosing an appropriate biased factor $a$. The biased factor can be expressed as the relationship between the average transmitted optical power $P_{t, o p t}$ and the total transmitted electrical power $P_{t, \text { elec }}$ with $a=P_{t, \text { opt }} / \sqrt{P_{t, \text { elec }}}$. The total transmitted electrical power can be obtained by $P_{t, \text { elec }}=\sum_{n=1}^{N-1} \mathrm{E}\left[x_{n}^{2}(t)\right]$, where $x_{n}(t)$ is the time-domain signal of the $n$-th subcarrier at time sample $t, \mathrm{E}[\cdot]$ denotes the expectation operation. The average transmitted optical power $P_{t, o p t}$ is the mean value of the time-domain signal after adding the DC bias $x_{D C}$ and therefore $P_{t, o p t}=x_{D C}[21]$.

\section{Optical Attocell Network Based on VLC Backhaul}

An optical attocell network is composed of an access network and a backhaul network. In order to guarantee access downlink transmission, each BS has been equipped with a group of VLC senders, i.e. visible-light LEDs. The access uplink transmission is separated with downlink by operating in infrared spectrum. Thus, infrared PDs embedded in BSs are used to receive data transmitted by UEs. In a VLCbased backhaul network, extra visible-light LEDs and PDs are required for backhaul transmission. Notice that the cost for replacing lamps with extra LED and PD chips is much 
(a)

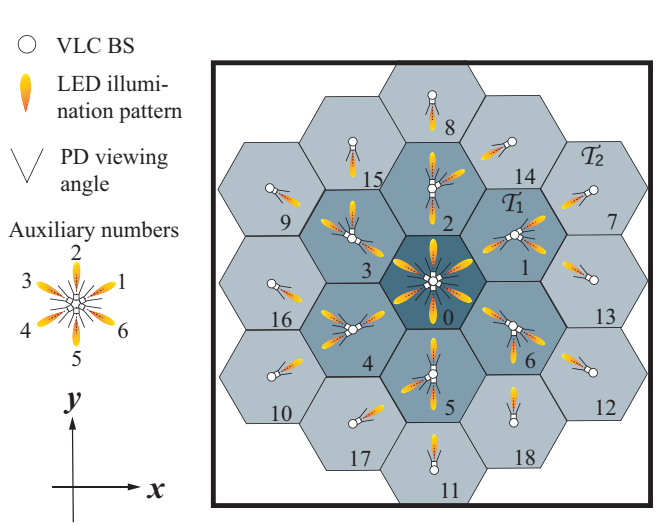

(b)

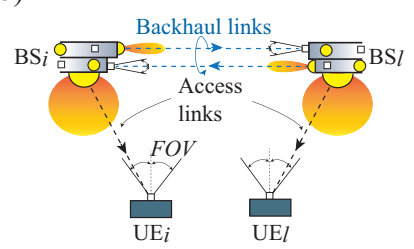

(c)

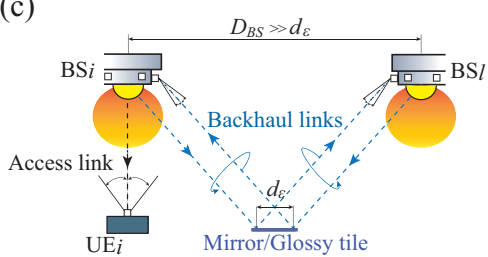

(d)

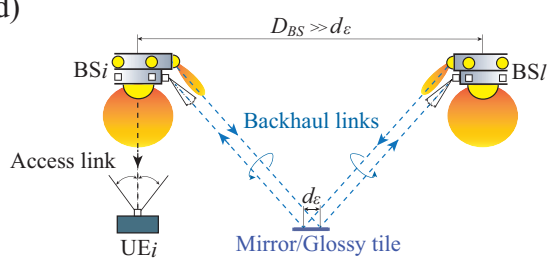

Fig. 3 (a) Layout of the optical attocell network with cell radius $R=2 \mathrm{~m}$ and horizontal orientation of auxiliary transceivers; (b) LOS (multi) configuration; (c) ma-nLOS (single) configuration; (d) ma-nLOS (multi) configuration

lower than that for replacing cables. Thus, VLC-based backhaul network is cost-efficient compared with wired backhaul approaches.

\subsection{Cellular system}

Hexagonal cells are considered in this paper as hexagons are proper approximations of circles and cover the whole floor without overlapping. Fig. 3(a) illustrates the layout of a twotier hexagonal cellular network with 19 cells. The length and width of the room are both $8 R$, where $R$ is the cell radius. Every BS is located in the center of one cell. All BSs are placed at the same height of $2.85 \mathrm{~m}$. Each BS has six auxiliary PDs for receiving backhaul data and uses downwardoriented LEDs or six auxiliary LEDs for backhaul transmission depending on the backhaul configuration. The horizontal projection of orientation of auxiliary transceivers, i.e. auxiliary LEDs and PDs, is also presented in Fig. 3(a). Each auxiliary transceiver is numbered based on its horizontal orientation. We denote $\mathrm{LEDs}$ of $\mathrm{BS}_{i}$ as $i-s j, j \in[0,1, \ldots, 6]$, where $s 0$ denotes downward-oriented LEDs. Similarly, auxiliary PDs of $\mathrm{BS}_{i}$ are denoted by $i-r j, j \in[1, \ldots, 6]$. The actual orientation of auxiliary transceivers depend on the backhaul link configuration and will be discussed later. Assuming that $\mathrm{BS}_{0}$ is the gateway, every $\mathrm{BS}$ is connected to the gateway directly or via multiple hops. Every intermediate BS decodes the received backhaul signal and forwards it to the next-hop BSs. In order to simplify the analysis, we assume a simple shortest-path routing scheme. Dijkstra algorithm is used for finding shortest paths as it is more time-efficient compared with other shortest-path routing algorithms like Bellman-Ford algorithm. The gateway communicates with its neighboring BSs, which are called tier-1 BSs $\mathcal{T}_{1}=\left\{\mathrm{BS}_{i}, i \in[1,2, \ldots, 6]\right\}$. Each tier-1 BS is responsible for backhaul data transmission with the gateway and two neighboring tier-2 BSs $\mathcal{T}_{2}=\left\{\mathrm{BS}_{i}, i \in[7,8, \ldots, 18]\right\}$. Fig. 3(a) shows the auxiliary transceivers used for backhaul communication based on the routing scheme. To simplify our analysis we consider that only downward-oriented LEDs and auxiliary LEDs shown in the figure are transmitting power. In more practical scenarios other transceivers could be used for different routing strategies or addressing shadowing problem.

\subsection{VLC backhaul configuration}

The LOS backhaul link configuration introduced in [16] uses horizontally placed auxiliary LEDs and PDs to ensure a direct LOS path. These auxiliary transceivers are embedded in the VLC light bulbs. Each auxiliary LED-PD pair enables unidirectional backhaul transmission from the LED transmitter to the PD receiver. In order to ensure bidirectional backhaul data transmission, each backhaul link requires two auxiliary LED-PD pairs having opposite transmitting directions. We assume full-duplex backhaul transmission. The self-interference between auxiliary LEDs and PDs mounted on the same BS is neglected due to following reasons: 1) Auxiliary LEDs have directive illumination pattern and the direct LOS path from the auxiliary LED to the auxiliary PD mounted on the same BS can be physically blocked [22]; 2) Multipath interference due to reflections via walls is not significant compared to the dominant intended LOS and mirror-aided non-LOS signal [17, 23]; 3) Multipath interference from ceiling reflection could be higher than other reflected components as lamps are assumed to be mounted on the ceiling. In this case, self-interference cancellation can be applied to retrieve the desired signal.

In some indoor scenarios, for instance recessed/embedded downlights or tray ceiling, there is no direct LOS path between two adjacent LED bulbs. As the quality of LOS link 
configuration depends on the LOS condition, obstructed LOS path and misaligned auxiliary transceivers will cause significant performance degradation. In order to address this issue, specular reflection can be used to replace a LOS path. Reflection from glossy surfaces and mirrors is directional and the reflectivity of practical mirrors is higher than diffuse surfaces. As discussed in [17], adding the first-order specular reflection to non-LOS channels increases the SNR by at least $10 \mathrm{~dB}$. Thus, the quality of mirror-aided non-LOS links is better than that of normal diffuse non-LOS links. In addition, mirror-aided non-LOS links using small mirrors are robust against multipath effect [17]. The shortest specular reflection path between two adjacent BSs is the path reflected by a mirror placed on the ceiling, i.e. mirrors oriented downwards, as upwards oriented lamps are normally placed close to the ceiling in order to illuminate a large area. The usage of this kind of configuration is limited as specular reflection from the ceiling could also be obstructed if the interior design is not possible for ensuring the LOS condition. Thus, we only discuss backhaul links using upward-oriented mirrors. Mirrors can be placed on the floor or tables. Although some specularly reflected paths could be (temporarily) obstructed by pedestrian or interior objects, this can be resolved by upper layers (for instance, by using routing protocols and hand-off schemes). For simplicity, we assume an empty room with mirrors on the floor. Mirrors are placed at the reflecting points, which are center points of edges of each hexagon as shown in the figure. The size of mirrors can be very small as long as the reflecting point is covered. Auxiliary PDs are oriented towards the corresponding reflecting points to maximize the received power. Downward-oriented LEDs, which are used for illumination and access transmission, can also be used to send backhaul signal to auxiliary PDs via specular reflections. However, different frequency bands should be allocated to different links in order to avoid interference. This configuration is low-cost but not spectral efficient. A second configuration is to use narrow-beam auxiliary LEDs oriented towards mirrors for backhaul transmission. This backhaul configuration benefits from high channel gain of direct mirror-aided non-LOS path. Although the infrared backhaul channel provides better performance as discussed in [16], direct radiation by infrared LEDs causes larger damage to human eyes than LEDs working in the visible range. This is due to the fact that the damage from infrared LEDs cannot be reduced by the natural aversion reflex of eyes as human eyes cannot perceive light at infrared range [24]. Thus, visible LEDs are used as auxiliary transmitters in our work. In following discussion, LOS configuration and mirror-aided non-LOS configuration using auxiliary LEDs are denoted by $L O S$ (multi) and ma-nLOS (multi), and mirror-aided nonLOS configuration using downward-oriented LEDs for backhaul transmission is denoted by ma-nLOS (single). Three link configurations are illustrated in Fig. 3(b), (c), and (d). Notice that in reality, the distances between LED and PD chips in the same BS are much smaller (a few centimeters) compared to the distance between two BSs. Thus, the distance between two reflecting points on the mirror $d_{\epsilon}$ shown in Fig. 3(c) and (d) is much smaller than the distance between two BSs $D_{B S}$. Without loss of generality, we assume that all LEDs and PDs of the same BS are located at the same point.

In order to avoid interference and multipath effect, the FOV of auxiliary PDs should be small. However, the relative orientation of auxiliary PDs with respect to mirrors/auxiliary LEDs determines the received power as seen in (1). In case of installation errors, auxiliary transceivers of some BSs may rotate against the vertical axis with a certain angle. Misaligned auxiliary PDs need enough viewing angle to receive the LOS signal or the signal from the one-bounce specular reflection. The configuration of FOV depends on layout of lamps as shown in Fig. 4 . The vector $\overrightarrow{n_{r}}$ and $\overrightarrow{n_{r}^{\prime}}$ denote orientation of perfectly aligned auxiliary PD and misaligned auxiliary PD, respectively. The maximum horizontal misalignment angle $\Delta \theta_{\max }=30^{\circ}$, as the auxiliary PD with $\Delta \theta_{\max }>30^{\circ}$ can be assigned to another LED (of a neighboring $\mathrm{BS}$ ). We consider the boundary cases when $\Delta \theta_{\text {max }}=30^{\circ}$ in Fig. 4. Auxiliary transceivers of LOS (multi) configuration shown in Fig. 4(a) are placed horizontally. The misaligned auxiliary $\mathrm{PD}$ of $\mathrm{BS}_{2}$ has to be able to see the auxiliary LED of its target $\mathrm{BS}_{0}$. The minimum FOV is equal to the maximum horizontal misalignment angle, i.e. $\Theta_{\max }^{b}=\Delta \theta_{\text {min }}$. Similarly, the maximum horizontal misalignment angle of ma-nLOS (multi) backhaul transceivers is also $30^{\circ}$ as shown in Fig. 4(b). The misaligned auxiliary $\mathrm{PD}$ of $\mathrm{BS}_{2}$ has to be able to receive the one-bounce specular reflection from the reflecting point on the mirror. Based on the geometry of mirrors and BSs, the minimum FOV is $\Delta \theta_{\text {min }}=\Theta_{\text {max }}^{b}=2 \arcsin \left(\frac{\frac{\sqrt{3} R}{2} \sin \frac{\Delta \theta_{\text {max }}}{2}}{\sqrt{H^{2}+\left(\frac{\sqrt{3} R}{2}\right)^{2}}}\right)$.

\section{Performance Analysis}

As visible light spectrum is used for access downlink and bidirectional backhaul links, the co-channel interference between access and backhaul links will be analyzed. The primary purpose of LED lamps is illumination. Thus, the transmitted power must take the illumination requirements into account. The aggregate data rate of optical attocell network using different backhaul configurations will be compared.

\subsection{Illuminance requirements}

In order to provide uniform illumination, wide-beam LEDs are normally used for indoor lighting. We assume that the semi-angle of downward-oriented LEDs $\Phi^{a}=60^{\circ}$. The primary purpose of these LEDs is illumination. Their trans- 
(a)

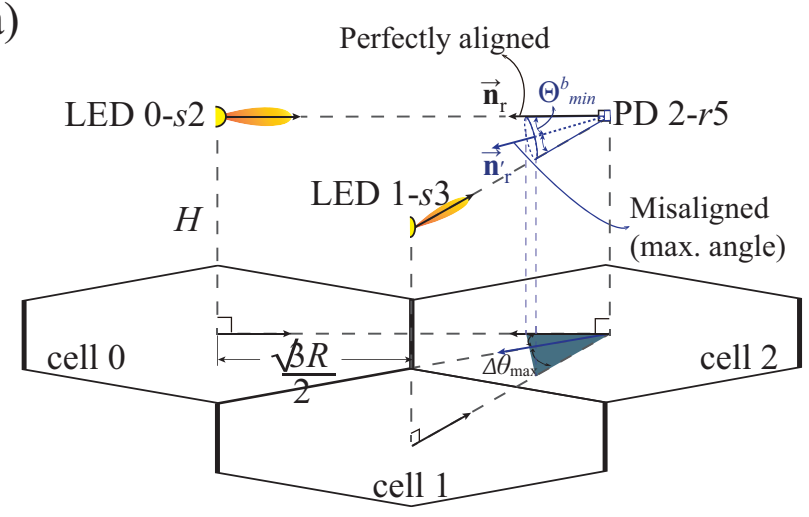

(b)

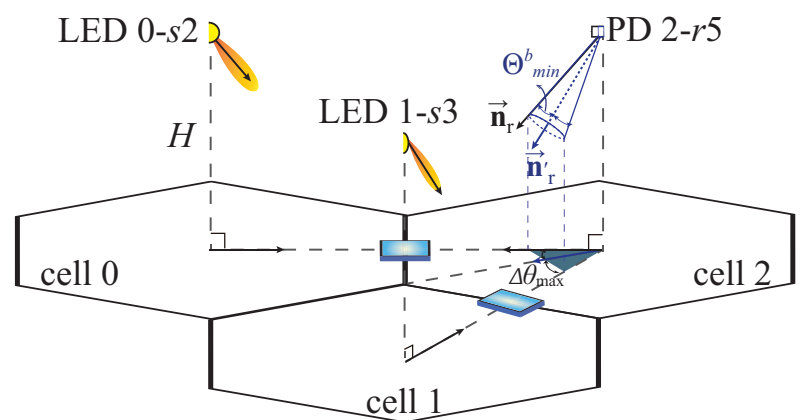

Fig. 4 Examples of misalignment of (a) LOS (multi) backhaul configuration and (b) ma-nLOS (multi) backhaul configuration

mitted power $P^{a}$ is determined by the illumination requirement. Recommended light levels for offices vary with activities [25]. Without loss of generality, we configure the transmitted power of downward-oriented LEDs providing average illuminance of $500 \mathrm{~lx}$ at desk height $(0.8 \mathrm{~m})$. In LOS (multi) backhaul configuration and ma-nLOS (multi) backhaul configuration, the primary purpose of the narrow-beam auxiliary LEDs is backhaul transmission. When auxiliary LEDs are added for backhaul transmission, the average illuminance should be limited under $7501 \mathrm{x}$, which is the maximum value required for activities in offices. In addition to the average illuminance requirement, eye-safety issues due to the concentrated illumination pattern must be considered. Although LEDs working in visible-light range are used for safety reasons as discussed in 3.2, direct and reflected light from narrow-beam visible LEDs may cause visual discomfort. Thus, a vertical eye illuminance threshold should be taken into account. Illuminance larger than this threshold may cause visual discomfort. There are large discrepancies among the existing and newly defined thresholds, which range from $875 \mathrm{~lx}$ to $3000 \mathrm{~lx}$ [26]. In this paper, we limit the transmitted power of auxiliary LEDs such that the maximum vertical illuminance on human eyes (assuming at height of $1.5 \mathrm{~m}$ ) is $2000 \mathrm{~lx}$. The maximum value of vertical illuminance on human eyes is measured at the point where the human can directly see the reflected light rays from mirrors. The illuminance values vary with transmitted power and the concentration of the light beam. The admission region of the ratio between transmitted power of downward-oriented LEDs and auxiliary LEDs $k=P_{o p t}^{b} / P_{o p t}^{a}$ and the semi-angle of auxiliary LEDs $\Phi^{b}$ can be found by drawing contour lines of maximum average illuminance and vertical illuminance threshold. Fig. 5 (a) and (b) show admission regions for LOS (multi) backhaul configuration and ma-nLOS (multi) backhaul configuration, respectively. Choosing the values of $\Phi^{b}$ and $k$ within these regions can fulfill the average illuminance requirement (less than $7501 \mathrm{x}$ ) and the vertical discomfort illuminance threshold (under 2000 lx). In Fig. 5 (a) it is shown that the vertical illuminance never exceeds $20001 \mathrm{x}$ and the average illuminance is larger than $7501 \mathrm{x}$ when $\Phi^{b}$ becomes large. It can be seen in Fig. 5 (b) that $P_{o p t}^{b}$ of the ma-nLOS (multi) backhaul configuration has a more strict limitation due to specular reflected light rays. When $\Phi^{b}$ is small, the concentrated light beam of auxiliary LEDs leads to very large vertical illuminance on human eyes, thereby limits the transmitted power of auxiliary LEDs. Increasing $\Phi^{b}$ allows larger transmitted power under the condition of vertical illuminance threshold. Unlike the decreasing trend of the contour line of average illuminance versus $\Phi^{b}$ in Fig. 5 (a), the 7501x) line of ma-nLOS (multi) configuration slightly decreases versus $\Phi^{b}$. This is likely due to the fact that auxiliary LEDs pointing towards mirrors on the floor has larger influence on average illuminance than horizontally placed auxiliary LEDs. Increasing $\Phi^{b}$ may reduce the maximum illuminance on the floor while increase the minimum illuminance, which leads to near constant contour lines of average illuminance versus $\Phi^{b}$. Regardless the backhaul configuration, increasing $k$ always leads to larger average and vertical illuminance when the value of $\Phi^{b}$ is fixed.

\subsection{SINR analysis of VLC backhaul}

Assuming all BSs are transmitting backhaul and access signals simultaneously, VLC backhaul links will interfere with access downlink transmission. Two frequency reused schemes, fully reused (FR) and in-band (IB) methods, have been discussed in [16]. With FR method access and backhaul links share the whole spectrum, while two orthogonal bands are used to transmit access and backhaul data with IB method. SINR of access and backhaul links depends on the backhaul configuration and the frequency reuse scheme. 
(a)

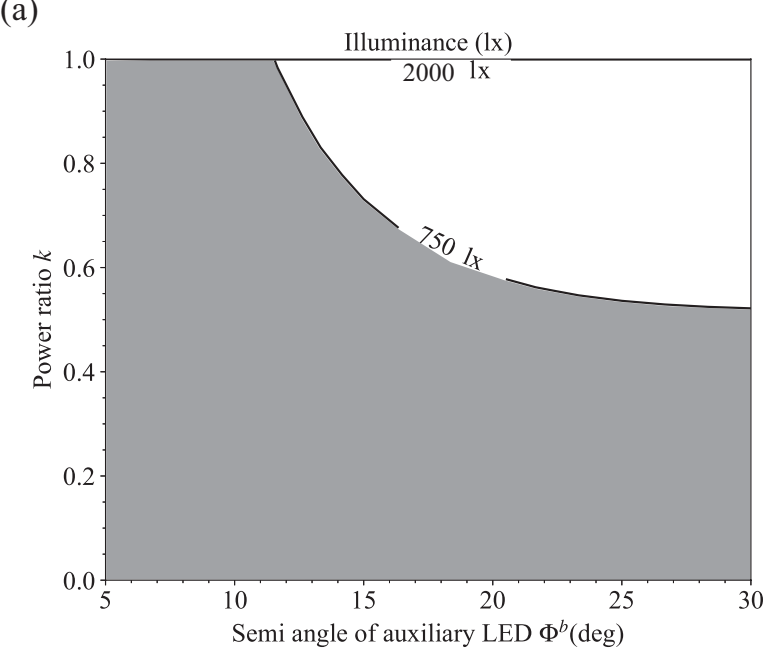

(b)

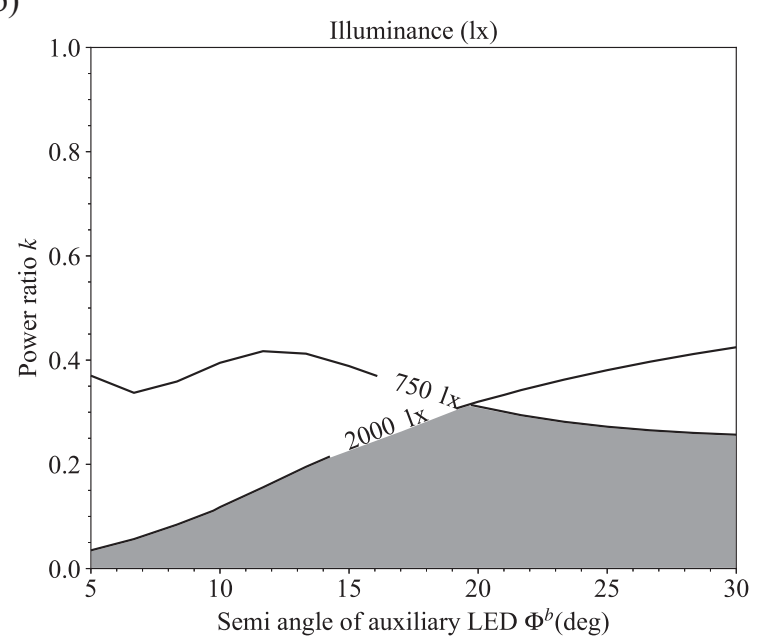

Fig. 5 Contour lines of the average illuminance requirement (750 1x) and the vertical illuminance threshold (2000lx) for (a) LOS (multi) backhaul and (b) ma-nLOS (multi) backhaul; shadowed areas are admission regions of power ratio $k$ and semi-angle $\Phi^{b}$

\subsubsection{Ma-nLOS (single) backhaul}

The purpose of downward-oriented LEDs is providing illuminance and access downlink transmission. When ma-nLOS (single) backhaul configuration is used, they are also used to send backhaul data. However, PDs of UEs detect access and backhaul signal sent from the same BS via the same LOS path. Similarly, auxiliary PDs also receive the power of access and backhaul signal sent from the same BS via the same mirror-obstructed LOS path. If the access link and backhaul link use the same spectrum and the equal amount of optical power is transmitted on both links, PDs detect very large interference came from the same path as the intended signal. Increasing the transmitted power of one link leads to lower SINR of the other link. In order to guarantee the performance, different orthogonal bands should be used to transmit access and backhaul data, i.e. IB method should be used. Thus, the SINR on the $n$-th subcarrier of an access link between a BS and a UE targeted to this BS is calculated as

$\gamma_{n}^{a-\mathrm{IB}}=\eta \frac{P_{\text {elec }, n}^{a} R_{P D}^{2}\left|G^{L O S}\right|^{2}}{\sigma_{n}^{2}+\sum_{i \in \mathcal{I}_{a}} P_{\text {elec }, n}^{a} R_{P D}^{2}\left|G_{i-s 0}^{L O S}\right|^{2}}$,

where $R_{P D}$ is the responsivity of PD in A/W, $\sigma_{n}=N_{0} B / N$ is the noise power on the $n$-th subcarrier, $N_{0}$ is the power spectral density (PSD) of AWGN channels, and $B$ is the bandwidth of OFDM signal, $G_{S}^{L O S}$ denotes the channel gain of the LOS path from a LED denoted by $s$ to the PD of the UE, the subscript is omitted for the path of the calculated access link, $P_{\text {elec }}^{a}$ denotes the transmitted electrical power for access data, which can be calculated by the optical power $P_{o p t}^{a}$ using the relationship in section 2.2. Here, we assume that electrical power is equally allocated on all modulated subcarriers, such that $P_{\text {elec }, n}^{a}=P_{\text {elec }}^{a} /(N-2)$. The second term in the denominator of the equation is the total electric power received from interfering sources. The interference set $\mathcal{I}_{a}$ consists of all BSs except for the tagged BS. The factor $\eta$ indicates the bandwidth efficiency loss caused by adding cyclic prefix (CP) at the end of each OFDM frame. Adding CP to OFDM frames can overcome inter-symbol interference (ISI) with simple equalization. The length of $\mathrm{CP}$ is chosen such that the $\mathrm{CP}$ duration is longer than 4 times the root-mean-square (RMS) delay spread of the channel, i.e. $T_{c p}=N_{c p} T_{s}=\left\lceil 4 \tau_{R M S} f_{s}\right\rceil \cdot T_{s}$, where $\lceil x\rceil$ is the least integer greater than or equal to $x, f_{s}$ is the sampling rate which is twice the signal bandwidth $B, T_{s}=1 / f_{s}$ is the sampling period, and $\tau_{R M S}$ is the RMS delay spread which is calculated using the simulated channel impulse response. Therefore the efficiency loss factor is calculated by

$\eta=\frac{N-2}{N-2+N_{c p}}=\frac{N-2}{N-2+\left\lceil 4 \tau_{R M S} f_{S}\right\rceil}$.

The IB method used in [15] divides the spectrum into two orthogonal bands and allocates them to access and backhaul transmission. All backhaul links share the same orthogonal band. However, backhaul data sent to different BSs interfere with each other when downward-oriented LEDs are used for backhaul transmission. As shown in Fig. 3(a), the gateway sends backhaul signal to six neighboring tier-1 BSs, each tier-1 BS sends backhaul downlink data to two tier-2 BS and uplink data to the gateway, and each tier-2 BS sends backhaul uplink data to the corresponding tier-1 BS. As auxiliary PDs are oriented towards different horizontal directions as shown in Fig. 3(a), interference from simultaneously transmitting backhaul links of their orienting BSs should be minimized. Thus, we divide the whole electric spectrum into six orthogonal bands which corresponds the maximum number of simultaneously transmitting backhaul links of one BS (the gateway). For simplicity, we allocate six orthogonal bands according to the number of auxiliary PD illustrated in Fig. 
3(a), i.e. an auxiliary $\mathrm{PD} i-r j$ of $\mathrm{BS}_{i}$ only detects interference signal intended to be sent to the auxiliary PD $l-r j$ of $\mathrm{BS}_{l}$. The SINR on the $n$-th subcarrier of a backhaul link between a downward-oriented LED and its targeted auxiliary PD is calculated as

$\gamma_{n}^{b-\mathrm{IB}}=\eta \frac{P_{\text {elec }, n}^{b} R_{P D}^{2}\left|G^{m a-n L O S}\right|^{2}}{\sigma_{n}^{2}+\sum_{i \in I_{b}} P_{\text {elec }, n}^{b} R_{P D}^{2}\left|G_{i-s 0}^{m a-n L O S}\right|^{2}}$

Similarly, $G_{s}^{m a-n L O S}$ denotes the channel gain of the mirroraided non-LOS path from the LED sender denoted by $s$ to the receiving auxiliary $\mathrm{PD}$, the subscript is omitted for the path of the calculated backhaul link, $P_{\text {elec }}^{b}$ denotes the transmitted electrical power for backhaul data. As downwardoriented LEDs are used for both access and backhaul data transmission, $P_{\text {elec }}^{b}=P_{\text {elec }}^{a}$. The interference set $\mathcal{I}_{b}$ consists of BSs transmitting backhaul data to an auxiliary PD with the same number of the targeted PD.

\subsubsection{Ma-nLOS (multi) backhaul}

When additional auxiliary LEDs are used for backhaul transmission, backhaul data is transmitted via mirror-obstructed LOS paths. If IB method is used, the SINR on the $n$-th subcarrier of access links is calculated as (2). The calculation of SINR of backhaul links is similar to (4). However, the interference set $\mathcal{I}_{b}$ for the ma-nLOS (multi) using the IB method becomes auxiliary LEDs with the same number as the tagged auxiliary LED. $P_{\text {elec }}^{b}$ is calculated by $P_{o p t}^{b}$ based on the illumination and eye safety requirements. As access and backhaul data is transmitted via different paths, FR method can be used. The SINR on the $n$-th subcarrier of access links using FR method is

$$
\begin{aligned}
& \gamma_{n}^{a-\mathrm{FR}}=\eta \frac{P_{\text {elec }, n}^{a} R_{P D}^{2}\left|G^{L O S}\right|^{2}}{\sigma_{n}^{2}+I}, \\
& I=\sum_{i \in \mathcal{I}_{a}} P_{\text {elec }, n}^{a} R_{P D}^{2}\left|G_{i-s 0}^{L O S}\right|^{2} \\
& +\sum_{i \in \mathcal{I}_{b}} \sum_{j \in \mathcal{H}_{i}} P_{\text {elec }, n}^{b} R_{P D}^{2}\left|G_{i-s j}^{L O S}\right|^{2},
\end{aligned}
$$

where $G_{S}^{L O S}$ denotes the channel gain of the LOS path from a downward-oriented LED or an auxiliary LED to the PD of the UE. The main difference between (2) and (5) is the total received interfering power. The interference set $\mathcal{I}_{a}$ of (5) consists of all BSs except for the tagged BS. The set $\mathcal{I}_{b}$ consists of all BSs and $\mathcal{A}_{i}$ consists of all power-transmitting auxiliary LEDs of $\mathrm{BS}_{i}$ (based on the routing scheme shown in Fig. 3(a)). The SINR on the $n$-th subcarrier of backhaul links can be calculated by

$$
\begin{aligned}
& \gamma_{n}^{b-\mathrm{FR}}=\eta \frac{P_{\text {elec }, n}^{b} R_{P D}^{2}\left|G^{m a-n L O S}\right|^{2}}{\sigma_{n}^{2}+I} \\
& I=\sum_{i \in \mathcal{I}_{a}} P_{\text {elec }, n}^{a} R_{P D}^{2}\left|G_{i-s 0}^{m a-n L O S}\right|^{2} \\
& +\sum_{i \in \mathcal{I}_{b}} \sum_{j \in \hat{\mathcal{A}}_{i}} P_{\text {elec }, n}^{b} R_{P D}^{2}\left|G_{i-s j}^{m a-n L O S}\right|^{2}
\end{aligned}
$$

where $G_{s}^{m a-n L O S}$ denotes the channel gain of the mirroraided non-LOS path from a downward-oriented LED or an auxiliary LED to the auxiliary PD. Here, $\mathcal{I}_{a}$ and $\mathcal{I}_{b}$ in $I$ both consist of all BSs. The set $\hat{\mathcal{A}}_{i}$ is a sub-set of $\mathcal{A}_{i}$ which excludes the targeted auxiliary LED.

\subsubsection{LOS (multi) backhaul}

Similar to ma-nLOS (multi) backhaul, FR and IB methods can be used for LOS backhaul configuration. SINR of access links can be calculated using the same equations presented in 4.2.1 and 4.2.2. Calculations of SINR of backhaul links are similar to that in the previous subsection. However, the channel gain of mirror-aided non-LOS paths should be replaced by that of LOS paths. As all BSs are placed at the same height, some LOS paths could be obstructed by BSs between them. Thus, there is no interference detected from these paths.

\subsection{Channel performance}

In this section, we assess the performance of backhaul configurations in terms of aggregate data rate of the network. Aggregate data rate is the sum of the data rates provided to all UEs in the network. The achievable data rates of a link is calculated using Shannon theorem $R=b \log _{2}(1+\gamma)$, where $b$ is the bandwidth allocated to the link, $\gamma$ is the SINR of the link. The end-to-end data rates of UEs in tier-1 and tier-2 cells are limited by backhaul data rates. If IB method is used, data rates of UEs in the gateway cell are affected by bandwidth allocation ratio $\delta=B_{a} / B$, where $B_{a}$ is the bandwidth allocated to access links. When multiple UEs are located in a cell, all UEs equally share the spectrum $B_{a}$. The sum of the data rates of these UEs achieves the maximum value when all UEs are located at a point with the maximum SINR, where is the center of the cell. It is clear that the maximum sum rate is equal to the achievable data rate of a single UE located at the cell center.

Due to asymmetric spatial distribution of SINR of access links in cells of tier- $2 \mathrm{BSs}$, the BSs in $\mathcal{T}_{2}$ are divided into two groups, which are $\mathcal{T}_{2} a=\left\{B S_{i}, i \in[7,8, . ., 12]\right\}$ and $\mathcal{T}_{2^{b}}=$ $\left\{B S_{i}, i \in[13,14, . ., 18]\right\}$, respectively. In order to calculate the aggregate data rate, we need to determine the bandwidth 
allocation ratio for different backhaul configurations using the IB method. As backhaul links cannot become bottleneck for data transmission to UEs, the data rate of each backhaul downlink $R_{\mathcal{T}_{i}}^{b-\mathrm{IB}}, i \in\left[1,2^{a}, 2^{b}\right]$ should be large enough to provide access data transmission to all downstream UEs, i.e. $R_{\mathcal{T}_{i}}^{a \text {-IB }}, i \in\left[1,2^{a}, 2^{b}\right]$. Based on the inequality $R_{\mathcal{T}_{1}}^{b-\mathrm{IB}} \geq R_{\mathcal{T}_{1}}^{a-\mathrm{IB}}+$ $R_{\mathcal{T}_{2} a}^{a-\mathrm{IB}}+R_{\mathcal{T}_{2 b}}^{a-\mathrm{IB}}$, the upper-bound of bandwidth allocation ratio of the gateway is calculated as in (7), where $\gamma_{n, \mathcal{T}_{i}}^{b \text {-IB denotes }}$ the SINR on $n$-th subcarrier of the backhaul link to $\mathcal{T}_{i}, i \in$ $\left[1,2^{a}, 2^{b}\right]$ BSs, $\gamma_{n, \mathcal{T}_{i}}^{a \text {-IB }}$ is the SINR on $n$-th subcarrier of the access link to UEs in $\mathcal{T}_{i}, i \in\left[0,1,2^{a}, 2^{b}\right]$ cells. Notice that $\gamma_{n, \mathcal{T}_{i}}^{a \text {-IB }}$ is calculated at the center point of the cell. The factor $\frac{1}{6}$ comes from six orthogonal bands used for backhaul data transmission to different neighboring BSs as discussed in section 4.2.1. As one tier-1 BS only serves two tier-2 BSs, $\delta_{\mathcal{T}_{1}}$ of tier-1 BSs is larger than that of the gateway. In a network using static frequency allocation, $\delta$ is fixed for every BS. In order to guarantee the access data transmission to all UEs, $\delta$ should be the bandwidth allocation ratio of the gateway cell, i.e. $\delta=\delta_{\mathcal{T}_{0}}$. Due to the illumination and eye safety requirements, the range of the optical power allowed to transmit backhaul data using auxiliary LEDs depends on the semi-angle of auxiliary LEDs as shown in Fig. 5. By taking different values of $\Phi_{b}$ and $k$, we observe that the maximum value of delta is obtained at maximum value of $k$ when $\Phi_{b}$ is fixed. We show the maximum values of $\delta$ with different values of semi-angle $\Phi^{b}$ in Fig. 6. The ratio $\delta$ of the ma-nLOS (single) backhaul configuration is very small and does not vary with $\Phi^{b}$. In ma-nLOS (multi) backhaul configuration, $\delta$ drops dramatically when $\Phi^{b}$ exceeds $10^{\circ}$. When $\Phi^{b}$ is larger than $20^{\circ}$, almost all bandwidth has to be allocated to backhaul links. The bandwidth ratio for LOS (multi) backhaul configuration is larger than that for the manLOS (multi) backhaul except for $\Phi^{b}=10^{\circ}$. We observe that the maximum value of $\delta$ is obtained at maximum value of $k$ shown in Fig. 5. Thus, at $\Phi^{b}=10^{\circ}$, ma-nLOS (multi) backhaul configuration is more power-efficient and achieves better performance than LOS (multi) backhaul configuration.

$\delta_{\mathcal{T}_{0}}=\frac{\frac{1}{6} \log _{2}\left(1+\gamma_{n, \mathcal{T}_{1}}^{b-\mathrm{IB}}\right)}{\frac{1}{6} \log _{2}\left(1+\gamma_{n, \mathcal{T}_{1}}^{b-\mathrm{IB}}\right)+\log _{2}\left(1+\gamma_{n, \mathcal{T}_{1}}^{a-\mathrm{IB}}\right)+\log _{2}\left(1+\gamma_{n, \mathcal{T}_{2} a}^{a-\mathrm{IB}}\right)+\log _{2}\left(1+\gamma_{n, \mathcal{T}_{2} b}^{a-\mathrm{IB}}\right)}$,

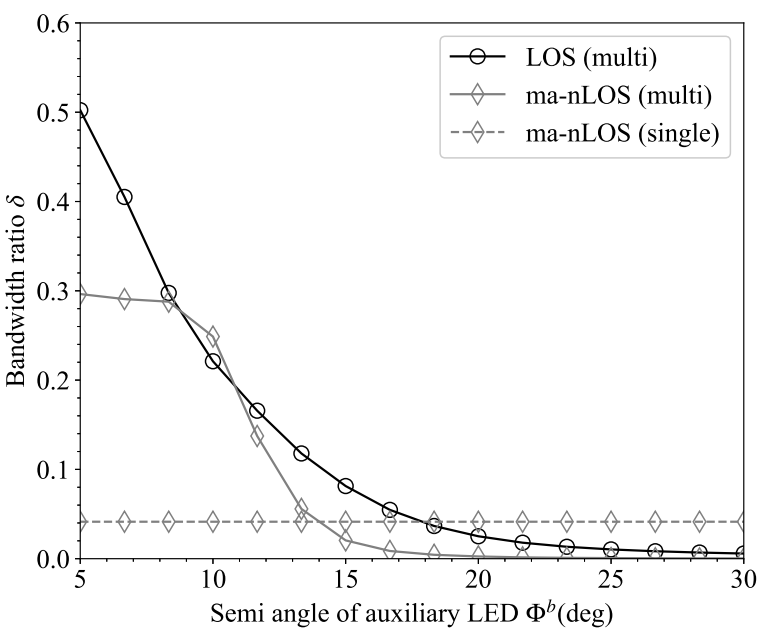

Fig. 6 Bandwidth allocation ratio $\delta$ of three backhaul configurations with different backhaul semi-angle $\Phi^{b}$; horizontal misalignment angle $\Delta \theta=0^{\circ}$; heights of UEs are $1.15 \mathrm{~m}$

Using $\delta$ values calculated in Fig. 5, the aggregate data rate of the network using IB method is $R=R_{\mathcal{T}_{0}}^{a \text {-IB }}+6\left(R_{\mathcal{T}_{1}}^{a-\mathrm{IB}}+\right.$ $\left.R_{\mathcal{T}_{2} a}^{a-\mathrm{IB}}+R_{\mathcal{T}_{2 b}}^{a-\mathrm{IB}}\right)$, where $R_{\mathcal{T}_{i}}^{a-\mathrm{IB}}=\xi \delta B \log _{2}\left(1+\gamma_{\mathcal{T}_{i}}^{a-\mathrm{IB}}\right), \xi=$ $(N-2) / 2 N$ is the bandwidth utilization factor of DCOOFDM. Similarly, the aggregate data rates of the network using FR method are calculated by replacing $R_{\mathcal{T}_{i}}^{a-\mathrm{IB}}$ with
Table 1 Simulation parameters

\begin{tabular}{c|c|c}
\hline Parameter & Symbol & Value \\
\hline Number of subcarriers & $N$ & 1024 \\
\hline Bandwidth & $B$ & $20 \mathrm{MHz}$ \\
\hline Noise PSD & $N_{0}$ & $1 \times 10^{-21} \mathrm{~A}^{2} / \mathrm{Hz}$ \\
\hline PD detecting area & $A_{r}$ & $1 \mathrm{~cm}^{2}$ \\
\hline PD responsitivity & $R_{P D}$ & $0.5 \mathrm{~A} / \mathrm{W}$ \\
\hline Reflectivity of mirrors & $\rho_{m}$ & 0.9 \\
\hline
\end{tabular}

$R_{\mathcal{T}_{i}}^{a-\mathrm{FR}}$, where $R_{\mathcal{T}_{i}}^{a-\mathrm{FR}}=\xi B \log _{2}\left(1+\gamma_{\mathcal{T}_{i}}^{a-\mathrm{FR}}\right)$. For comparison, the aggregate data rate of a network using backhaul links with unlimited capacity is also calculated. The mirrors used in our simulations are assumed aluminium-coated mirrors. Without loss of generality, the reflectivity of mirrors is obtained by taking the average of $\Phi_{e}(\lambda) \rho(\lambda) / \Phi_{e}(\lambda)$ over the visible light spectrum, where $\Phi_{e}(\lambda)$ is the spectral density of a commercial blue LED with yellow phosphor at wavelength $\lambda$ and $\rho(\lambda)$ is the reflectivity of aluminium-coated mirrors at $\lambda$. Parameters are shown in table 1 . Results of aggregate data rates are shown in Fig. 7. The network using backhaul with unlimited capacity achieve maximum $1.45 \mathrm{Gbps}$. The network using ma-nLOS (single) backhaul configuration using IB method only achieve $0.07 \mathrm{Gbps}$. For the rest backhaul configurations which use auxiliary LEDs for backhaul transmission, it is clearly shown that the performance highly depends on the FOV of auxiliary LEDs. The aggregate 
data rate drops when $\Phi^{b}$ increases. LOS (multi) backhaul configuration with FR method outperforms other backhaul configurations. It can achieve almost the same performance as backhaul with unlimited capacity when $\Phi^{b}$ is very small. However, the performance degrades dramatically when increasing $\Phi^{b}$. When IB method is used, the performance of LOS (multi) backhaul configuration degrades due to low spectral efficiency. The case of ma-nLOS (multi) backhaul is exactly the opposite: using IB method can achieve better performance than using FR method. When IB method is used and $\Phi^{b}$ is between $7^{\circ}$ and $11^{\circ}$, this backhaul configuration performs as good as LOS (multi) backhaul configuration using the same frequency reuse scheme. When $\Phi^{b}>13^{\circ}$ the ma-nLOS (single) configuration out performs ma-nLOS (multi) using IB method. If FR method is used for ma-nLOS (multi) backhaul, the network performance is the worse than the ma-nLOS (single) configuration when $\Phi^{b}$ is larger than $11^{\circ}$. The aggregate data rate of the network slightly increases by minimizing the semi-angle of auxiliary LEDs.

In the above analysis, horizontal misalignment angle $\Delta \theta=0^{\circ}$ is assumed. This ideal case may not be guaranteed in some scenarios, for instance, one of the BSs rotates against the vertical axis due to installation errors or cleaning. We calculate network aggregate data rates in the above mentioned misalignment scenario and show in Fig. 8. We assume that the bandwidth allocation ratio $\delta$ calculated by (7) does not change. The data rate ratio $R_{m i s}(\Delta \theta) / R(0)$ indicates the robustness of the backhaul configuration against misalignment. Here, $R(0)$ is the aggregate data rate when all auxiliary transceivers are perfectly aligned $\left(\Delta \theta=0^{\circ}\right)$, and $R_{\text {mis }}(\Delta \theta)$ is the aggregate data rate when rotating auxiliary transceivers (both PDs and LEDs) of the misaligned BS with horizontal misalignment angle $\Delta \theta$. Auxiliary transceivers of other BSs remain the same orientation. The positive value of $\Delta \theta$ means rotating auxiliary transceivers (both PDs and LEDs) of the misaligned BS anti-clockwise. It is shown in Fig. 8 that the LOS (multi) configuration is vulnerable to misalignment than the ma-nLOS (multi) configuration regardless the frequency reuse scheme. The data rate ratio $R_{\text {mis }}(\Delta \theta) / R(0)$ of networks using the LOS (multi) configuration suddenly drops when $\Delta \theta$ of auxiliary transceivers of $\mathrm{BS}_{0}$ is greater than $\pm 13^{\circ}$ as shown in Fig. 8 (a). This is likely due to the fact that the SINR of LOS backhaul links dramatically decreases due to misalignment and backhaul links become bottleneck when $\Delta \theta> \pm 13^{\circ}$. When the horizontal misalignment angle $\Delta \theta$ of $\mathrm{BS}_{0}$ is very large, for instance, $\Delta \theta= \pm 20^{\circ}$, networks using ma-nLOS (multi) and IB method can achieve higher aggregate rates $(0.25 \mathrm{Gbps})$ than other configurations (lower than $0.1 \mathrm{Gbps}$ ). A misaligned tier-1 or tier-2 BS has almost no effect on ma-nLOS (multi) configuration. It is also shown that $R_{m i s}(\Delta \theta) / R(0)$ is symmetric around $\Delta \theta=0^{\circ}$ when $\mathrm{BS}_{0}$ is misaligned. However, it is not the case for other misaligned BSs shown in Fig. 8 (b), (c), and (d). This is caused by unsymmetrical placement of auxiliary transceivers of tier-1 BSs. The simulation results reveal that mirror-aided nonLOS configurations are more suitable for indoor application having higher requirements of stability.

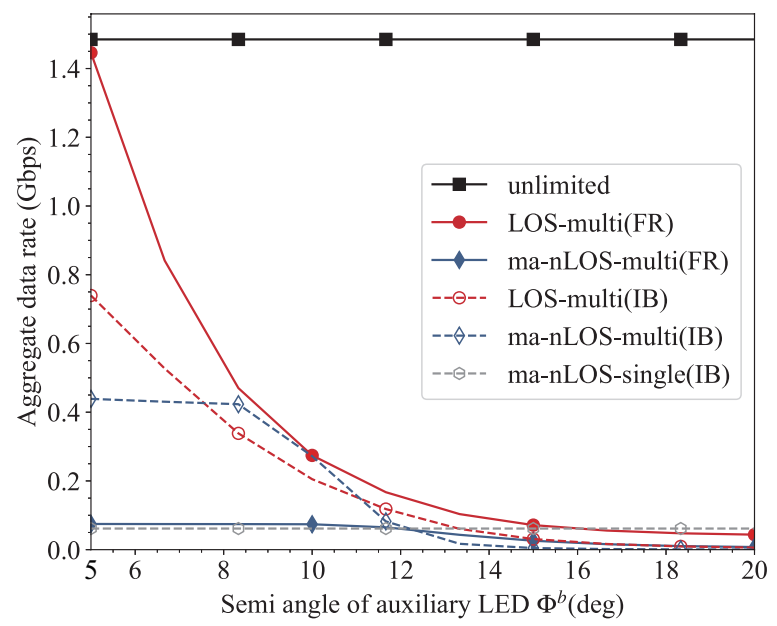

Fig. 7 Aggregate data rates of optical attocell networks using different backhaul configurations; horizontal misalignment angle $\Delta \theta=0^{\circ}$; heights of UEs are $1.15 \mathrm{~m}$

\section{Conclusion}

The purpose of this study is to analyze the performance of optical attocell networks using VLC backhaul links taking into account illumination and eye safety requirements. A LOS backhaul configuration (LOS (multi)) and two mirror-aided non-LOS configurations (ma-nLOS (single) and ma-nLOS (multi)) are analyzed and compared. The transmitted power of auxiliary LEDs should be limited due to indoor illumination requirements and eye safety issues. The ma-nLOS (multi)configuration has a more strict limitation on the auxiliary transmitted power. Bandwidth allocation ratio for networks using IB frequency reused scheme is calculated. The ma-nLOS (multi) configuration requires larger bandwidth for backhaul transmission than the LOS (multi) configuration in order to avoid throughput bottleneck. Aggregate data rates of optical attocell networks using different backhaul configuration are compared. A FR frequency reuse scheme is also considered. It is shown that LOS (multi) configuration using the FR scheme outperforms other backhaul configurations. The ma-nLOS (multi) configuration using IB method can achieve almost good performance as the LOS (multi) configuration using the same frequency reuse strategy. Results also reveal backhaul configurations using auxiliary LEDs need narrowbeam auxiliary transmitters to guarantee the performance of the network. Finally, performance comparison of backhaul 


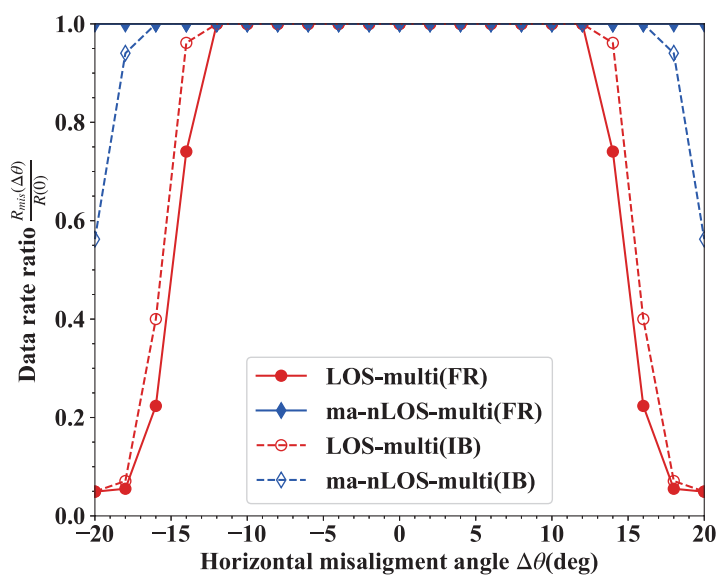

(a) Misaligned BS0

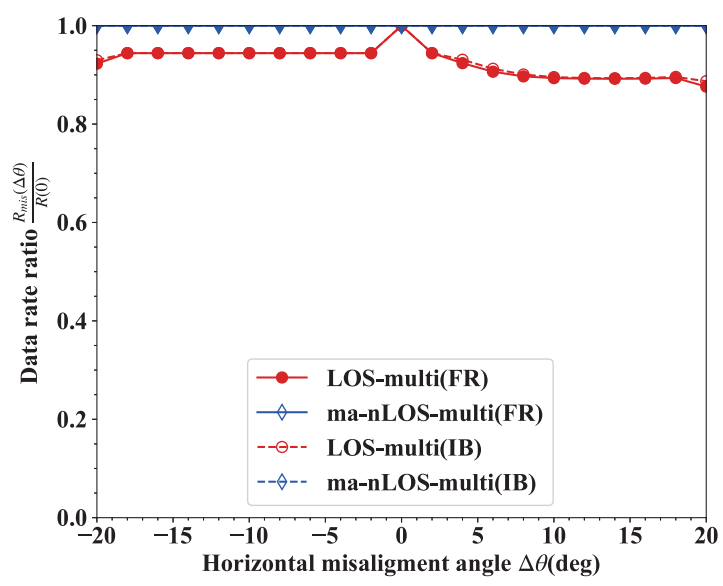

(c) Misaligned BS7

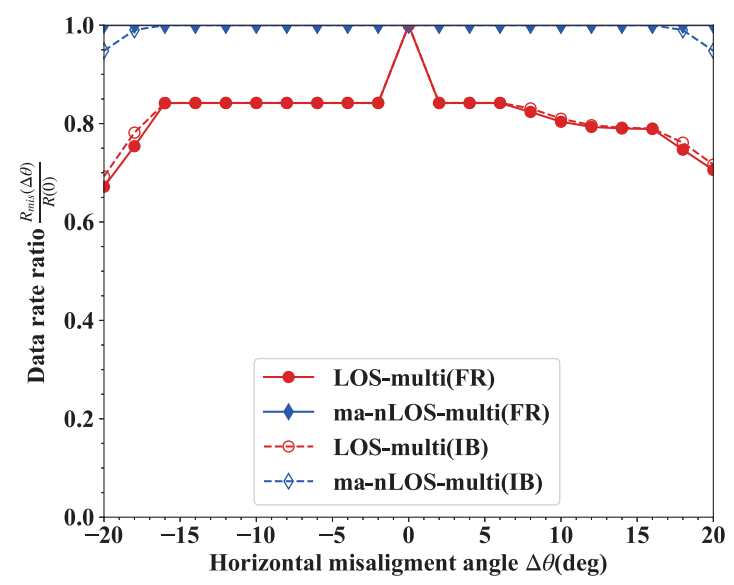

(b) Misaligned BS1

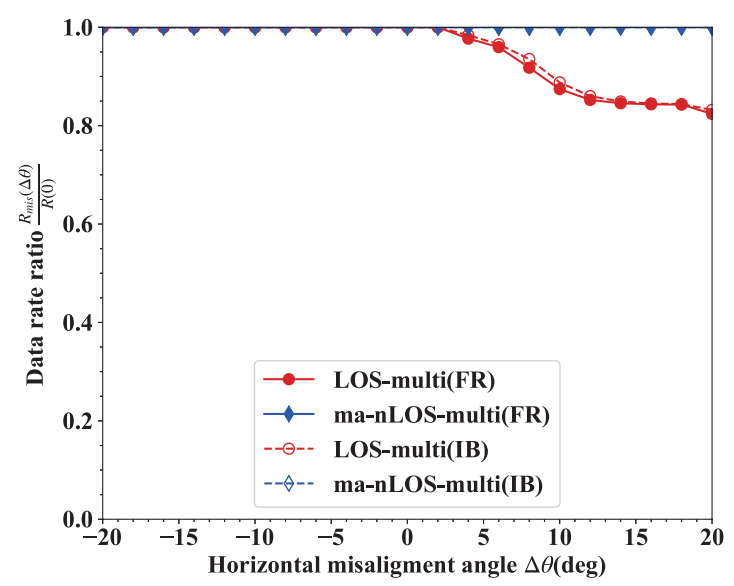

(d) Misaligned BS14

Fig. 8 Data rate ratio $R_{\text {mis }}(\Delta \theta) / R(0)$ of networks using two backhaul configurations when rotating auxiliary transceivers of (a) $\mathrm{BS}_{0}$ (gateway), (b) $\mathrm{BS}_{1}\left(\mathcal{T}_{1}\right),(\mathrm{c}) \mathrm{BS}_{7}\left(\mathcal{T}_{2}^{a}\right)$, and $(\mathrm{d}) \mathrm{BS}_{14}\left(\mathcal{T}_{2} b\right)$ against the vertical axis; $\Phi^{b}=5^{\circ}$; heights of UEs are $1.15 \mathrm{~m}$

links using a gateway with misaligned auxiliary transceivers shows that the LOS (multi) configuration is more vulnerable to misalignment.

\section{References}

1. H. Burchardt, N. Serafimovski, D. Tsonev, S. Videv, H. Haas, IEEE Communications Magazine 52(July), 98 (2014)

2. A. Sevincer, A.B. Member, M.B. Member, IEEE Communications Surveys \& Tutorials 15(4), 1620 (2013)

3. D.K. Borah, A.C. Boucouvalas, C.C. Davis, S. Hranilovic, K. Yiannopoulos, EURASIP Journal on Wireless Communications and Networking pp. 1-28 (2012)

4. V. Jungnickel, M. Uysal, N. Serafimovski, T. Baykas, E. Ciaramella, Z. Ghassemlooy, R. Green, H. Haas, P.A. Haigh, V.P.G. Jimenez, F. Miramirkhani, M. Wolf, IEEE Conference on Standards for Communications and Networking (CSCN) pp. 106-111 (2015)

5. H. Elgala, R. Mesleh, H. Haas, IEEE Communications Magazine 49(9), 56 (2011). DOI 10.1109/MCOM.2011.6011734

6. H. Kazemi, H. Haas, IEEE International Conference on Communications (2016). DOI 10.1109/ICC.2016.7511475
7. C. Chen, D.A. Basnayaka, H. Haas, Journal of Lightwave Technology 34(1), 137 (2016). DOI 10.1109/JLT.2015.2511015

8. I. Stefan, H. Burchardt, H. Haas, IEEE International Conference on Communications pp. 3825-3829 (2013). DOI 10.1109/ICC.2013.6655152

9. T. Komine, M. Nakagawa, IEEE Transactions on Consumer Electronics 49(1), 71 (2003)

10. Y. Wang, N. Chi, Y. Wang, L. Tao, J. Shi, IEEE Photonics Technology Letters 27(2), 197 (2015). DOI 10.1109/LPT.2014.2364955

11. D. Karunatilaka, F. Zafar, V. Kalavally, R. Parthiban, IEEE Communications Surveys \& Tutorials 17(3), 1649 (2015). DOI 10.1109/COMST.2015.2417576

12. J. Liu, B. Zhao, L. Geng, Z. Yuan, Y. Wang, in IEEE International Symposium on Power Line Communications and Its Applications (2011). DOI 10.1109/ISPLC.2011.5764448

13. L. Feng, R.Q. Hu, J. Wang, P. Xu, Y. Qian, IEEE network (2016). DOI 10.1109/MNET.2016.1500236RP

14. T. Wu, T.S. Rappaport, C.M. Collins, IEEE Microwave Magazine (2015). DOI 10.1109/MMM.2014.2377587

15. H. Kazemi, M. Safari, H. Haas, in IEEE ICC 2017 Optical Networks and Systems Symposium (2017)

16. H. Kazemi, M. Safari, H. Haas, IEEE Transactions on Wireless Communications (2019). DOI 10.1109/TWC.2018.2883465 
17. Y. Wu, P. Audenaert, M. Pickavet, D. Colle, Photonic Network Communications 38(38), 151 (2019). DOI 10.1007/s11107-01900841-3

18. C. Chen, S. Videv, D. Tsonev, H. Haas, Journal of Lightwave Technology 33(19), 3986 (2015). DOI 10.1109/JLT.2015.2458325

19. F.R. Gfeller, U. Bapst, Proceedings of the IEEE 67(11), 1474 (1979). DOI 10.1109/proc.1979.11508

20. J. Kahn, J. Barry, Proceedings of the IEEE 85(2), 265 (1997). DOI 10.1007/978-1-4615-2700-8

21. J. Armstrong, B.J.C. Schmidt, IEEE Communications Letters 12(5), 343 (2008). DOI 10.1109/LCOMM.2008.080193

22. H. Yang, A. Pandharipande, in Industrial Electronics Conference (IEEE, 2013), pp. 6075-6080. DOI 10.1109/IECON.2013.6700133

23. O. Narmanlioglu, R.C. Kizilirmak, F. Miramirkhani, M. Uysal, IEEE Photonics Journal 9(3), 1 (2017). DOI 10.1109/JPHOT.2017.2708746

24. R.S. Office, Laser Safety Mannual (2007)

25. European Committee for Standardization, Light and lighting Lighting of work places - Part 1: Indoor work (2002). DOI http://www.din.de

26. J.Y. Suk, Building and Environment 148, 107 (2019). DOI 10.1016/j.buildenv.2018.10.058 